\title{
Fibromialgia: Construção e Realidade na Formação dos Médicos
}

\section{Fibromyalgia: Construction and Reality in Medical Education}

\author{
Rejane Leal Araújo ${ }^{(1)}$
}

\section{PROBLEMAS ATUAIS NOS MODELOS DE CONSTRUÇÃO DAS DOENÇAS}

A construção conceitual da síndrome da fibromialgia (FM) como doença, das suas superposições ou co-morbidades e das suas relações com a fragmentação do conhecimento no atual contexto sociohistórico constituem um grande desafio para o exercício do médico. Sua identificação segue os critérios de classificação do American College of Rheumatology (ACR-1990), os quais foram desenvolvidos com o propósito de analisar resultados interinstitucionais da avaliação de grupos populacionais semelhantes, embora também possam ser utilizados para diagnósticos individuais ${ }^{(1)}$.

O estudo dos atuais modelos para as construções científicas da FM, e outras síndromes expressas por sintomas, estimula o desenvolvimento de outras percepções, absorvidas com a inserção do terceiro fator, além do sujeito e do objeto, que é o estado do conhecimento ${ }^{(2)}$. O rótulo fibromialgia permitiu acalmar os médicos que passaram a dispor de um termo biomédico para se comunicarem e explicarem aos pacientes, que se sentiram satisfeitos com a validação de seus sintomas como reais e não como simulados.

Goldenberg $^{(3)}$ afirma que, apesar do número crescente de publicações, pouco progresso tem sido feito para a compreensão ou tratamento desse distúrbio e seu conceito permanece controverso, incluindo aqueles que continuam a questionar sua existência e sua utilidade diagnóstica.

A prevalência da FM de 0,5 a $4 \%$ nos países industrializados $^{(4)}$, acrescida pela superposição das síndromes semelhantes, mas com linguagens diferentes, implica em gastos socioeconômicos e desgastes individuais, familiares e sociais, para entender e explicar problemas de saúde, rotulados como síndromes em sua multiplicidade de conotações biomédicas, genéticas, psicológicas, cognitivas e socioculturais.

\section{ESTUDO DAS RELAÇÕES SOCIOHISTÓRICAS: EPISTEMOLOGIA, EDUCAÇÃO E SAÚDE}

As propostas epistemológicas atuais para transformação do modelo de construção do conhecimento científico determinam mudanças na dinâmica e na qualidade dos modos de pensar, pois excluem as neutralidades e inserem a participação contextual, evoluem do estático para o processual e estudam a complexidade com o emaranhado de ações, interações e retroações.

Deste modo, o exercício do pensamento complexo implica em transformações na conceituação dos binômios como: pensamento estático/processual, dissociado/complexo, saúde/doença, unidade/todo, unicausal/multicausal,

1. Médica: Fellow em Reumatologia, UVA-USA, Mestre em Educação pela Universidade Federal de Santa Catarina (UFSC) e Professora de Medicina da Universidade do Sul de Santa Catarina (UNISUL).

Endereço para correspondência: Rejane Leal Araújo, e-mail: rejane@rotolo.trix.net 
biomédico/biopsicossocial. Com esse interesse, Araújo ${ }^{(5)}$ estudou a relação entre o modelo atual de construção dos distúrbios como a FM com os modelos epistemológicos fragmentados que dominaram o século XX, conhecidos como cartesianos, ainda hegemônicos nas teorias e práticas atuais da educação e da saúde. Destaca que a fragmentação do modelo da educação médica americana de Flexner ${ }^{(6)}$, seguido pelo Brasil, priorizou as teorias e as práticas restritas ao biomédico, separadas em disciplinas, ensinadas por médicos especialistas. Este modelo, que domina as construções diagnósticas atuais, se limita aos sintomas do domínio de cada especialista, com baixo grau de representatividade para a composição do todo do indivíduo.

Apesar das diferenças nos rótulos diagnósticos especializados, os pacientes têm em comum o comportamento da vigília constante (ansiedade/angústia), são, em sua grande maioria, do gênero feminino e tendem a se apresentar com uma variedade de sintomas crônicos associados a distúrbios de humor (depressão) e distúrbios do sono (insônia). Os sintomas, como a dor, estudados e interpretados por especialistas, ficam restritos às construções de um saber delimitado: sabem muito de um determinado assunto, mas muito pouco do restante. Com base nesses e em outros dados, Araújo $^{(5)}$ propõe a tríade da depressão, insônia e dor/fadiga como representativa da dinâmica integrada pelos fatores físicos biomédicos, psicoemocionais, comportamentais e socioculturais para justificar o significado e a expressão das diferentes síndromes crônicas, co-mórbidas ou em overlap com a FM. Seu estudo fundamentou-se na categoria epistemológica, o estilo de pensamento (thought style $)^{(7)}$, descrita pelo médico e filósofo polonês Ludwig Fleck (1935). O estilo de pensamento foi definido como um perceber dirigido com a correspondente elaboração intelectiva e objetiva do percebido. Comparado à categoria paradigma (Kuhn, 1962), consiste em uma determinada atitude, e também ao tipo de execução que a consuma (integra a teoria e a prática).

Diferentes autores ${ }^{(8-11)}$ utilizam estilo de pensamento como categoria para documentar os problemas que surgem pela aplicação do modelo cartesiano utilizado no século passado para a construção do conhecimento científico. O estudo e a aplicação dos diferentes elementos que compõem o estilo/coletivo de pensamento permitiram constatar que apesar da uniformidade curricular do curso de graduação, os médicos se distanciam pelas suas formações especializadas durante as residências médicas. Os especialistas estudam e pensam assuntos relativos a seus compartimentos especializados, com suas linguagens próprias, exercícios fragmentados e freqüentam congressos restritos a suas especialidades. Esse modelo acentua a formação reduzida a assuntos especializados, compondo coletivos de pensamento dissociados, que constroem estilos de pensamento com incongruência de linguagens. Os médicos não são formados para entender a unicidade de cada indivíduo e construir modelos que expliquem a complexidade dos doentes e as propostas da interação biopsicossocial na saúde.

TABELA 1

Fibromialgia e Síndromes Funcionais Somáticas FragmentaÇão CiENTÍFICA

\begin{tabular}{|c|c|c|}
\hline ESPECIALISTA & SINTOMA & SÍNDROME \\
\hline REUMATOLOGISTA & DOR CRÓNICA/DIFUSA & FIBROMIALGIA \\
\hline INFECTOLOGISTA & FADIGA CRÓNICA & SÍND. FADIGA CRÓNICA \\
\hline ORTOPEDISTA & DOR LOCALIZADA & $\begin{array}{l}\text { SÍND. DOR. REG. } \\
\text { MIOFACIAL }\end{array}$ \\
\hline PSIQUIATRA & DEPRESSÃO & DEPRESSÃO MAIOR \\
\hline NEUROLOGISTA & DORES DE CABEÇA & $\begin{array}{l}\text { SÍND. CEFALÉIAS / } \\
\text { ENXAQUECA }\end{array}$ \\
\hline $\begin{array}{l}\text { ESPECIALISTA DO } \\
\text { SONO }\end{array}$ & INSÔNIA & DESORDENS DO SONO \\
\hline GASTROENTEROLOGISTA & $\begin{array}{l}\text { EVACUACCÕES } \\
\text { FREQUENTES }\end{array}$ & $\begin{array}{l}\text { SÍNDROME CÓLON } \\
\text { IRRITÁVEL }\end{array}$ \\
\hline MÉDICO DO TRABALHO & $\begin{array}{c}\text { DOR/FADIGA, } \\
\text { ATIVIDAD. REPETIDAS }\end{array}$ & LER - DORT \\
\hline
\end{tabular}

Na Tabela 1 são apresentadas diferentes construções diagnósticas superpostas, como a FM, a síndrome da fadiga crônica, a síndrome do cólon irritável, a dor miofacial regional e a depressão, fruto das construções por estilos de pensamento especializados e fragmentados. São restritas à expressão isolada dos sintomas, que não abrangem a relação biomédica com os aspectos sociocognitivos, embora esses possam representar o significado, ou seja, a essência comum a muitos dos pacientes. Omite a importância do perfil cognitivo de entender, reagir ou conviver com seus sintomas e problemas.

\section{MODELO DE CONSTRUÇÃO DA SÍNDROME DA FIBROMIALGIA}

A FM, ao exibir como critérios objetivos os pontos dolorosos, estabelece dificuldades de precisão que envolvem o subjetivo da expressão do paciente e o da interpretação médica. Clauw ${ }^{(12)}$ defende que pelo menos metade dos indivíduos que recebem o diagnóstico clínico da FM não 
preenche sua definição e que aproximadamente $25 \%$ das pessoas com desordens sistêmicas inflamatórias podem ter positivos os critérios do ACR para essa síndrome. Esse autor ressalta que os critérios de classificação nunca tiveram a intenção de serem aplicados com o propósito de diagnóstico individual. Comenta que os pontos dolorosos medem não apenas a sensibilidade, mas também o modo de lidar com o estresse, influenciado por diferentes fatores como: gênero feminino, aumento da idade, deficiência do condicionamento físico e desordens do humor. Rao e Clauw ${ }^{(13)}$ conferiram o nome de síndromes funcionais somáticas (SFS) para classificar grupo semelhante de desordens relativas aos pacientes com as características relatadas anteriormente.

Contextualizar a dinâmica da FM como a ruptura de um continuum construído pelo paciente com seu modo de lidar com estresses abre perspectivas que implicam nos diferentes significados das doenças e suas responsabilidades individuais e sociais, como nos diagnósticos de lesão por esforço repetitivo ou distúrbio osteomuscular relacionado ao trabalho (LER/DORT). Essa diversidade desafia a postura médica frente às normas estabelecidas pela perícia e concessão de benefícios públicos. Sobre esse assunto, Aronowitz, em seu livro Making Sense of Illness ${ }^{(14)}$, publica um inovador documento histórico científico sobre as controvérsias e de como as doenças podem mudar de significado ao longo dos tempos pelas mudanças de suas hipóteses etiológicas.

Crofford e Clauw ${ }^{(15)}$, em análise sobre os efeitos após uma década dos critérios ACR-1990, salientam que os mesmos enfocam apenas a dor e desprezam outros sintomas importantes da FM incluindo fadiga, distúrbios cognitivos, distúrbios do sono e angústia psicológica. Esses autores destacam uma visão mais ampla que inclui esses sintomas e as síndromes comumente associadas (síndrome da fadiga crônica, síndrome do cólon irritável e depressão) $)^{(16)}$ para concluir que esses critérios falham em apreender a essência da FM. Num estudo sobre a relação do temperamento e resposta ao estresse em crianças com o diagnóstico da síndrome primária da fibromialgia juvenil (JPFMS), Conte $^{(17)}$ obteve como resultados que crianças e adolescentes com JPFMS, assim como seus pais, apresentavam diferenças quando comparados a outros grupos. Aqueles com diagnóstico de JPFMS demonstravam maior instabilidade do temperamento, níveis maiores de depressão e ansiedade, menor ajuste familiar e maior sensibilidade à dor. Seus pais também referiam aumento nos níveis de ansiedade e depressão e menor capacidade de ajuste psicológico. Conte defendeu a perspectiva psicobiológica e destacou a interação dos riscos individuais como a instabilidade do temperamento, a sensibilidade à dor, a vulnerabilidade ao estresse, o contexto, o ajuste e as psicopatologias familiares como fatores contribuintes para explicar a composição dessa síndrome da dor e sua severidade. Os dados que antecedem, relativos às histórias de vida e à representação de seus significados, podem se constituir em fatores diferenciais para o entendimento das síndromes em estudo.

Giesecke e Clauw ${ }^{(18)}$ reconhecem que os indivíduos que preenchem os critérios do ACR de classificação para FM não compõem um grupo homogêneo. Defendem a hipótese de existirem distintos subgrupos entre os pacientes com FM, que podem ser identificados pelos padrões de sensibilidade/dor, humor e cognição. Para eles, parece existir um grupo de pacientes com FM que exibe extrema sensibilidade sem associação com fatores psicocognitivos; um grupo intermediário e um terceiro grupo, nos quais os fatores relacionados ao humor e a cognição influenciam no relato dos sintomas. Esses são os pacientes que referiam alta sensibilidade à dor, apresentavam altos índices de depressão e ansiedade, emitiam seu quadro como catastrófico, além de ter baixa capacidade para o controle da dor. Tais resultados podem ter implicações terapêuticas quanto à escolha de analgésicos ou dos tipos de antidepressivos e psicotrópicos.

\section{PERSPECTIVAS BIOPSICOLÓGICAS ATUAIS}

A construção conceitual da FM e da sua co-morbidade com a depressão maior (DM) apóia-se no modelo biológico que pesquisa substâncias neurotransmissoras (serotonina, norepinefrina, substância $\mathrm{P}$ ), assim como na sua interação com o modelo psicossocial que relaciona a tristeza e a dificuldade de adaptação, demonstradas nas respostas de dramatizar ou não saber se ajudar.

Aparentemente, existe uma separação das múltiplas redes de processamento dos sintomas como a dor e a depressão. Os níveis dos sintomas da depressão não atuam diretamente na magnitude da ativação das áreas cerebrais associadas com a dimensão sensorial da dor (córtices somatossensoriais primária e secundária). No entanto, os sintomas da depressão ou a presença da depressão parecem estar associados com a ativação das regiões cerebrais relacionadas com o processamento da dor afetiva (amígdala e ínsula anterior contralateral). Dados da literatura sugerem a ínsula anterior como atuante na interação das experiências sensoriais e afetivas ${ }^{(4)}$.

Esses achados podem se estender às outras condições com dores crônicas que tenham um elemento central ca- 
racterizado por hiperalgesia/alodínia como a síndrome do cólon irritável, a lombalgia e a vulvodínia. Sugerem que este fenômeno de ativação neural no processamento da dor possa ser notado também em condições periféricas e clássicas como a osteoartrite e a artrite reumatóide.

\section{OBJETO FRONTEIRA: SUA IDENTIFICAÇÃO PARA INTERAGIR O BIOPSICOSSOCIAL}

Ao se propor o estudo da relação dos fatores psicossociais como influentes ou determinantes dos modos de construção e expressão dos diferentes processos que alteram a saúde, há que se modificar o modelo tradicional das consultas médicas que separam em pedaços a avaliação do indivíduo. Excluir das consultas a contextualização do problema do paciente e a relação processual com suas his- tórias de vida e as construções sociocognitivas é eliminar a percepção das essências desses processos, cujas construções não conseguem ser integralmente explicadas pelo conhecimento biomédico. A categoria boundary object (objeto fronteira), foi proposta por $\operatorname{Star}^{(19)}$, para estandardizar os métodos e como atividade central para a tradução e comunicação entre os diferentes pontos de vista. Araújo ${ }^{(20)}$ propôs a utilização da tríade de sintomas da depressão, insônia e dor como objeto fronteira para integrar trabalho multidisciplinar entre os diferentes profissionais que estudam a FM e as síndromes funcionais somáticas. Seu trabalho foi apresentado no 2004 ACR Economic, Social and Psychological Impact of Arthritis.

A abertura para esses novos aspectos resultará em futuras construções sociointeracionistas mais elaboradas, hoje classificadas como interdisciplinares que buscam a transdisciplinaridade (Figura 1).

Figura 1

\begin{tabular}{|c|c|c|c|c|c|}
\hline \multicolumn{2}{|c|}{ MULTIDISCIPLINARIDADE - fragmentação } & \multicolumn{2}{|c|}{$\begin{array}{c}\text { OBJETOS FRONTEIRA } \\
\text { (representados por setas) }\end{array}$} & \multicolumn{2}{|c|}{ INTERDISCIPLINARIDADE } \\
\hline Psicologia & Antropologia & Psicologia & Antropologia & Psicologia & Antropologia \\
\hline & & & & \multicolumn{2}{|c|}{ Conhecimento interdisciplinar emergente } \\
\hline $\begin{array}{c}\text { Medicina } \\
\text { (neurologia, } \\
\text { reumatologia, } \\
\text { infectologia, } \\
\text { etc.) }\end{array}$ & Fisioterapia & $\begin{array}{c}\text { Medicina } \\
\text { (neurologia, } \\
\text { reumatologia, } \\
\text { infectologia, } \\
\text { etc.) }\end{array}$ & Fisioterapia & $\begin{array}{c}\text { Medicina } \\
\text { (neurologia, } \\
\text { reumatologia, } \\
\text { infectologia, } \\
\text { etc.) }\end{array}$ & Fisioterapia \\
\hline
\end{tabular}

Consciente da interação entre os fatores sensoriais e afetivos motivacionais no resultado da dor, o médico precisa estudar sua relação com os fatores culturais regionais, com as condições e satisfações da vida, a legislação trabalhista, assistencial e pericial. Vale avaliar, portanto, a geração e a persistência da amplificação dos sintomas como a dor em sua relação mais estreita com a formação cognitiva do indivíduo e seus desgastes físicos pela incapacidade de lidar com determinados problemas psicossociais. Os estudos biomédicos, como as substâncias neuroendócrinas e os estudos de imagem e sua relação com outros achados co-mórbidos, continuam sendo importantes. Porém, a inserção e a articulação dos outros eventos psicossociais podem resultar em novos modelos para entender os processos hoje classificados como doenças.

Esse é o grande desafio para o profissional com formação, prática e construções biomédicas: construir um ver formativo e perceber as interações dos fatores biopsicossociais. Valorizar a multiplicidade dos fatores: a susceptibilidade genética e sua interação com os fatores sociocognitivos, biológicos, psicoemocionais, familiares, do gênero e ambientais, assim como buscar a integração dos diferentes sistemas para compor a visão do todo, implicam em transformações conceituais curriculares e na formação dos professores. São processos lentos que implicam em mudanças conceituais dos modos de pensar e exercitar o pensamento, necessárias para a conquista da harmonia dos diagnósticos e das práticas periciais. Portanto, interagir o estudo dos diferentes sistemas auxilia na percepção da importância da transformação epistemológica que amplia o modo de pensar, disjuntivo e redutor, para o encontro do complexo com a real interpretação do significado dos modelos da expressão corporal. 
Hadler $^{(21,22)}$ comenta sobre a organização de um sistema que com intervenções médicas prejudiciais à saúde reduzem a qualidade de vida. Destaca o uso da FM como rótulo de doença que incapacita, e que essa construção social da mesma gera litígios e discussões epistemológicos atuais sobre as fronteiras entre suas realidades e verdades.

A inserção curricular do estudo da epistemologia e a aplicação das categorias estilo/coletivo de pensamento e objeto fronteira estimulam o desenvolvimento da abordagem sistêmica e da capacidade crítica dos assuntos pertinentes à educação e à saúde. Estende a condição espectadora dos professores e estudantes para a de participantes no processo da construção interdisciplinar e interação dos sistemas com o conhecimento científico (Figura 2).

\section{REFERÊNCIAS}

1. Criteria for the Classification and Diagnosis of the Rheumatic Diseases. Primer on the Rheumatic Diseases: $12^{\underline{a}}$ Ed, Atlanta, Arthritis Foundation, 631-648, 2001.

2. Fleck, L: La Génesis y el Desarollo de un Hecho Científico. Madrid, Alianza, 1986.

3. Goldenberg, DL: A Fibromialgia Uma Década Depois: O Que Aprendemos? JAMA Brasil - J Am Med Assoc 8: 3507-3515, 2000. Tradução de: Fibromyalgia Syndrome a Decade Later: What Have We Learned? Arch Intern Med 159: 777-785, 1999.

4. Giesecke T, Clauw DJ et al: The Relationship Between Depression, Clinical Pain, and Experimental Pain in a Chronic Pain Cohort. Arthritis Rheum 52: 1577-1584, 2005.

5. Araújo RL: Doenças: Construção e Realidade na Formação dos Médicos. Dissertação de Mestrado em Educação e Ciência, Universidade Federal de Santa Catarina, 2002.

6. Flexner A: Medical Education: A Comparative Study. Macmillian, 1925.

7. Fleck L: Genesis and Development of a Scientific Fact. Chicago: The University of Chicago Press, 1979.

8. Löwy I: The Strength of Lose Concepts, History of Science, 30: 371-395, 1993.

9. Löwy I: Between Bench and Bedside, Harvard University press, 1996.

10. Löwy I: Ludwik Fleck and the Notion of "boundary object". Mimeo do I Seminário Internacional sobre Filosofia da Saúde, Florianópolis, 1996.

11. Delizoicov D: Sobre a Produção do Conhecimento Científico (Editorial) Caderno Catarinense de Ensino de Física, Florianópolis, 13: 178-276, 1996.

12. Clauw DJ: Fibromyalgia and Diffuse Pain Syndromes. In:
Figura 2

Prática InTEgrada: Um Requisito PARA a COMUNIDADE MÉDICA

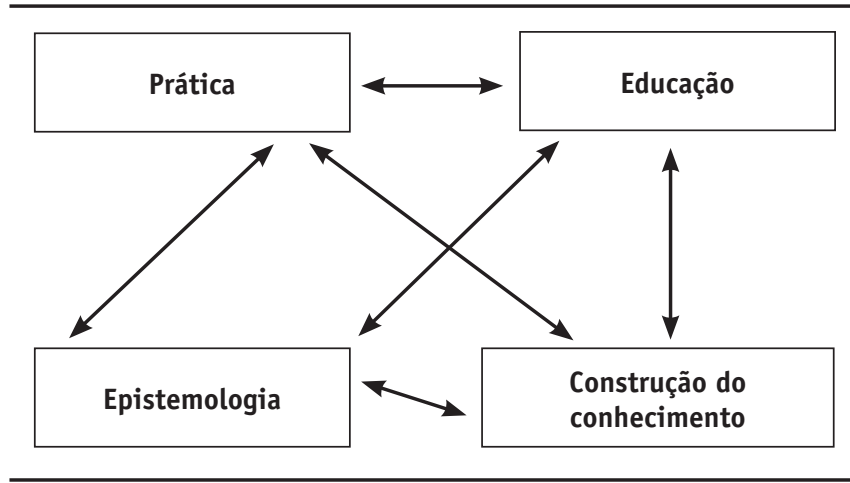

Primer on the Rheumatic Diseases. $12^{a}$ ed, Atlanta, Arthritis Foundation: 188-193, 2001.

13. Rao SG, Clauw DJ: The Management of Fibromyalgia, San Diego, Cypress Bioscience, 2004.

14. Aronowitz RA: Making Sense of Illness: science, society, and disease. Cambridge: Cambridge University Press, 1998.

15. Crofford, LJ, Clauw DJ,. Where are we a decade after the American College of Rheumatology classification criteria were developed? Editorial: Arthritis Rheum, New York, 46: 11361138, 2002.

16. Engleberg NC: Chronic fatigue syndrome. In: Mandel et al. Principles and Practice of Infectious Diseases. $15^{\underline{a}} \mathrm{ed}$, Philadelphia, Churchill Livingstone, 529-1534, 2000.

17. Conte PM, Walco GA, Kimura Y: Temperament and Stress Response in Children with Juvenile Primary Fibromyalgia Syndrome, Arthritis Rheum 8, 10: 2923-2930, 2003.

18. Giesecke T, Clauw DJ et al: Subgrouping of Fibromyalgia Patients on the basis of Pressure-Pain Thersholds and Psycological Factors. Arthritis Rheum 48: 2916-2922, 2003.

19. Star SL, Griesemer JG: Institutional Ecology, Translations and Boundary Objects: Amateurs and professionals in Berkeley's Museum of Vertebrate Zoology, 1907-39. Social Studies of Science, 19: 387-470, 1989.

20. Araújo RL: The Symptoms of Depression, Insomnia and Pain/ Fatigue as a Boundary Object in Multidisciplinary Work. Arthritis Rheum 50, 9: S 48, 2004.

21. Hadler MN: Occupational Muskuloskeletal Disorders, Lippincott Williams \& Wilkins, $3^{\underline{a}}$ ed, 2005.

22. Hadler M N: The Last Well Person (How to Stay Well Despite), The Health-Care System McGill-Queen's University Press, 2004. 